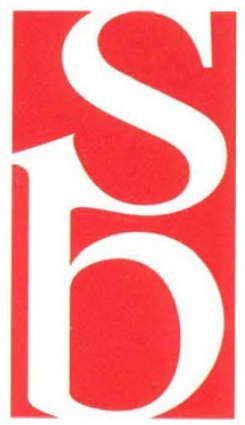

\title{
The Changing Structure of Biology
}

Why start another journal?

It is easy to sympathize with those who ask "Why yet another?" Every month seems to see the launch of a new journal aimed at the structural community, while the flood of fresh structures swells with their every issue. But while there is no shortage of vacant niches for someone with a structure to publish, Nature Structural Biology will be something different.

Of course, a venture of this kind would be impossible were it not for the rapid growth of interest in the field. The concatenation of technical advances that have made this possible has been rehearsed so often that it needs no repetition; those who want more details need look no further than pages 8-10 of this issue, where Stephen Burley explains the situation in 'Forward to the Fundamentals'.

In 1993 alone, biologists were enthralled by structures of the MHC class II HLA-DR1 dimer, the nuclear pore complex, the myosin S1 domain, the complex of the TATA-binding protein with DNA and the $\alpha$ subunit of transducin, among many others. There are greater heights to be scaled in the near future: the structures of GroEL, the hammer-head ribozyme, the ADP ribosylation factor, F1 ATPase and penicillin synthase, for example. And on everyone's wish list must be the ribosome, although that vast complex will take a little longer.

In 1994, one new structure will on average be determined every day and, if that trend merely continues to the beginning of the next century, the year 2000 will see 2,800 structures not at present known. The first objective of Nature Structural Biology, then, will be to provide a forum, at first on paper but eventually electronically, for the rapid dissemination of these results.

There is always a rush of excitement at the first sight of a structure. The molecule in question may previously have been well known, but it will have been known only as a phenotype, a gene sequence or even a band on a gel. Almost invariably, the structure moves researchers a step closer to understanding how the molecule goes about its business. But scarcely less frequently, it raises more questions than it answers, emphasizing that the static image of the molecule is rarely an end in itself, but rather a beginning of comprehension.

With structures becoming ever more easily determined, the emphasis will shift from description, an important initial phase in any area of scientific endeavour, to the dissection of function. What are the forces that drive the association of proteins with their substrates? How do proteins fold to the native state both in vitro and in vivo? What are the factors that determine the stability of proteins? How do proteins turn chemical energy into force? The structural basis of molecular function will be the bread and butter of Nature Structural Biology.

The array of tools for this purpose is becoming ever more impressive. One need look no further than this year's Nobel prize for chemistry, awarded to the discoverers of site-directed mutagenesis and the polymerase chain reaction. And new techniques are no doubt being developed even now. Technical advances that are likely to change the face of the field and pioneering applications of existing techniques will certainly be featured in the pages of Nature Structural Biology. The paper by Wade et al. on pages 65-69 illustrates the power of brownian motion simulations, for example.

With an understanding of the structural basis of function comes the possibility of interven- 
tion. Proteins and nucleic acids can be engineered to be more or less stable, more efficient enzymes can be built and enzymic substrate specificity can be altered. Drugs can be designed to interfere with crucial steps in the progression of disease. Indeed, the idea of a bidentate, 'hydra-headed' inhibitor is raised in the paper by Mattos et al. on pages 55-58. Clearly the insights gained from structural studies will have important medical and industrial ramifications that will be featured in Nature Structural Biology.

Although proteins directly affect almost every biological process, life would not be possible in the absence of nucleic acids, carbohydrates and lipids. The versatility of RNA, in particular, continues to surprise. In vitro experiments have produced RNA molecules that can bind a wide range of ligands and catalyse reactions not known to be RNA-catalysed in nature.

An understanding of the way in which ribozymes work was once a fascinating molecular curiosity with implications for the beginnings of life on Earth. But its ramifications now seem likely to be even wider, as both splicing and translation may well be catalysed by RNA. Nature Structural Biology will naturally pay particular attention to developments in these areas; indeed, the paper on the Tetrahymena ribozyme on pages 13-17 (discussed on pages 5-7) takes another step towards understanding that molecule's action.

It is the aim of this new journal to disseminate new information about biologically relevant molecular structures as quickly as possible, and to the widest possible audience. It is then possible to hope that the importance of the advances reported will itself generate general interest. But primary research will be backed up by informed comment and opinion both on issues affecting workers in the field and on the wider implications of the results. The intention is that Nature Structural Biology will be essential reading for all with interests in molecular structures and in molecular biology in general.

The question of the relationship between this journal and its parent journal Nature needs clarifying. The past four issues of Nature demonstrate that its interest in structural biology continues unabated. Nature Structural Biology is not intended to take structural biology away from Nature, but rather to publish much of what Nature itself cannot accommodate. Between them, the two journals will in future be publishing even more in this field, in the hope of being able to accommodate the ever-increasing volume of excellent research.

Clearly there is a link between the two journals extending beyond the common name. How will this work? Nature Structural Biology is at once a part of Nature and separate from it. Within the general framework of an agreed editorial policy (which includes the same criteria of excellence for both journals), Nature Structural Biology will benefit from less restrictive rules on the length of manuscripts, while bringing them to the attention of a more specialist audience than Nature. But would-be contributors must make their own guesses about the relative merits of sending their manuscripts to one journal or the other.

The day-to-day link between the two journals is that they share traditions and facilities. More practically, when Nature is unable to publish an otherwise excellent manuscript for lack of space, it may offer to pass the manuscript (with the accompanying referees' reports where applicable) to its sister journal. (It will be for authors to decide whether to avail themselves of that offer.) Nature will also give its general readership an account of what Nature Structural Biology has just published. The overall objective is more faithfully to reflect the interest of this important field.

These are indeed exciting times for structural biology. The years to come will see a structure-led revolution in our understanding of the molecular processes that underpin life on Earth, and even perhaps the way in which that life began. The consequences of this revolution will reverberate across all of biology. How lucky we are that it will be in our own lifetimes!

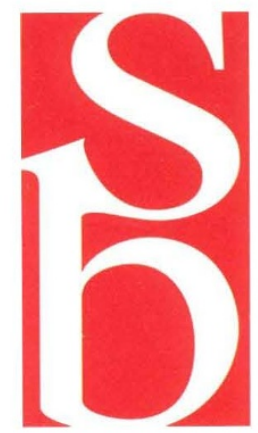

